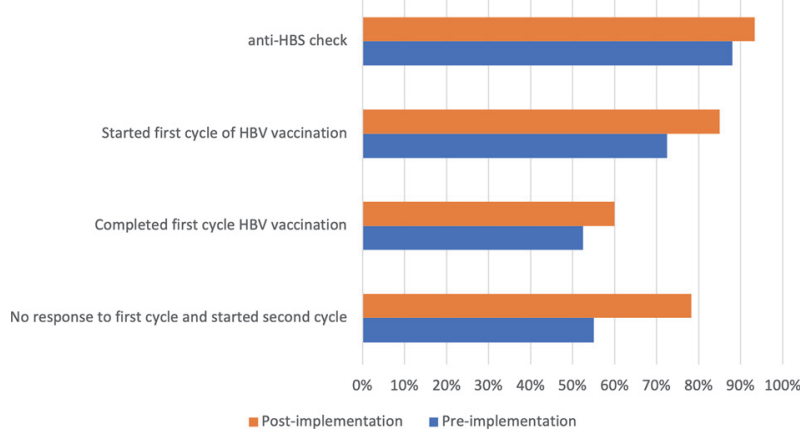

Abstract IDDF2021-ABS-0190 Figure 1

Methods A cross-sectional study of all eligible LT recipients from 2008 to 2020 in a leading transplant centre in Singapore was conducted to determine the vaccine response in HBV-naïve subjects. A standardised workflow was devised to identify barriers in vaccination and response monitoring. Transplant coordinators and pharmacists assisted physicians to identify and prescribe CDC-recommended vaccinations using a template, which include Engerix $40 \mathrm{mcg}$ of three doses, followed by rechecking anti-HBs titre one month after. A second 3 -dose Engerix regimen was given if anti-HBs remains $<10$ $\mathrm{mIU} / \mathrm{mL}$. Non-responder is defined as failure to achieve protective anti-HBs titre after completing two cycles.

Results Of the 279 LT recipients, we excluded patients not on follow-up and patients with anti-HBc positivity. 75 patients were included. Prior to vaccination implementation, 9/75 $(12 \%)$ were not checked for anti-HBs. Of the 66 checked, 40 required vaccination. $29 / 40(72.5 \%)$ were started on the first cycle, and 21/40 (52.5\%) completed. 11/20 (55\%) patients were initiated on the second cycle. Post workflow implementation, 40 needed vaccination. 34/40 (85\%) were started on the first cycle and 24/40 (60\%) completed. 18/23 (78.3\%) were started on a second cycle. 3/4 (75\%) were non-responders (IDDF2021-ABS-0190 Figure 1). Our implementation coincided with the COVID-19 pandemic where more teleconsultation was used. COVID-19 vaccination was also prioritised over HBV vaccination. We postulate that these results would be further improved once physical consultations resumed.

Conclusions The development of a standardised workflow can lead to improvement in anti-HBs testing and compliance to vaccination post-LT. These findings may be useful for other patients who are on long-term immunosuppression. Continual efforts from the multidisciplinary team are required to ensure the sustainability of effect.

\section{IDDF2021-ABS-0203 A SCREENING MODEL FOR OBSTRUCTIVE SLEEP APNEA ON THE BASIS OF FATTY LIVER DISEASE-RELATED PARAMETERS}

\footnotetext{
${ }^{1,2}$ Yang-Bor Lu*, 1,2Yu-Chieh Weng, 1,2Yung-Ning Huang, ${ }^{3}$ Hsiung-Ying Huang, ${ }^{4}$ PeiTing Cheng, ${ }^{5}$ Hui-Shan Hsieh, ${ }^{6,7}$ Ming-Shao Tsai. ${ }^{1}$ Department of Digestive Disease, Xiamen Chang Gung Hospital, Xiamen, China; ${ }^{2}$ Hepatobiliary and Pancreatic Unit, Xiamen Chang Gung Hospital, Xiamen, China; ${ }^{3}$ Department of Pulmonary and Critical Care Medicine, Sleep center, Xiamen Chang Gung Hospital, Xiamen, China; ${ }^{4}$ Formosa Biomedical Technology Corp., Taipei, Taiwan; ${ }^{5}$ Department of Otolaryngology-Head and Neck Surgery, Sleep center, Xiamen Chang Gung Hospital, Xiamen, China; ${ }^{6}$ Department of Otolaryngology-Head and Neck Surgery, Chiayi Chang Gung Memorial Hospital, Chiayi, Taiwan; ${ }^{7}$ College of Medicine, Chang Gung University, Taoyuan, Taiwan
}

10.1136/gutjnl-2021-IDDF.103
Background/Aims Obstructive sleep apnea (OSA) among patients with nonalcoholic fatty liver disease (NAFLD) has an emerging increased trend, thus noninvasive screening methods are urgently needed to screen for OSA risk in these patients. Therefore, we aimed to screen them while conducting an office-based survey of hepatic steatosis. The routine hepatic check-up methods, such as controlled attenuation parameter (CAP) and hepatic steatosis index (HSI) in patients with or without OSA, are investigated and developed the screening model to detect OSA.

Methods The medical records of all adult patients (aged $\geq 18$ years) receiving routine liver sonography examination from June 2017 to June 2020 with completed CAP, polysomnography, and HSI data in our hospital were retrospectively reviewed.

Results A total of 59 patients were included in this study. Among them, 62.7\% (37/59) and 74.6\% (44/59) (detected by HSI and CAP, respectively) had NAFLD, and 78\% (46/59) were diagnosed with OSA- based on standard in-laboratory polysomnography. Binary logistic regression models showed that sex (male, odds ratio 4.17 [95\% CI: 1.76-298.92]), body mass index (BMI) (> 24.8, odds ratio 1.42 [95\% CI: 1.09 1.86]), and HSI (> 38.3, odds ratio 1.17 [95\% CI: 1.02 1.36]) significantly screening OSA risk, in descending order of odds ratio. Multivariate analysis showed that male sex, BMI, and HSI independently screen OSA and their combination best screen for OSA risk (sensitivity $=78 \%$; specificity = $85 \%$; and positive and negative predictive values $=95 \%$ and $52 \%$, respectively; area under the curve $=0.85$ ).

Conclusions Our result suggests that HSI has better screening performance than CAP. A combination of male, BMI, and HSI proposed here provides a noninvasive and rapid screening tool for OSA risk. The model can be employed while patients receive routine hepatic check-ups in clinical practice. That can be used to efficiently screen for at-risk patients, and thus facilitate earlier detection and timely treatment intervention.

\section{Clinical Gastroenterology}

\begin{tabular}{l|l}
\hline IDDF2021-ABS-0005 & NUTRITION SUPPORT TEAM FOR \\
& INTESTINAL FAILURE PATIENTS ON \\
& PARENTERAL NUTRITION: IMPROVING \\
& MACRO-AND-MICRONUTRIENTS INTAKE
\end{tabular}

Ho Yan Terry Ting*, Kai Hong, Edmond Luk, Pui Sze, Grace Lui, Hiu Yan, Sharon Chan, Ho Kin, Ivan Mak, Kai Hong, Alessandro Leung, Yuk Ying Lam, Chi Yan Wong, Wai Yin, Sally Luk. Nutrition Support Team, North District Hospital, Hospital Authority, Hong Kong

\subsection{6/gutjnl-2021-IDDF.104}

Background A nutrition support team (NST) is a multidisciplinary team of physicians, pharmacists, nurses and dietitians, who are responsible for managing patients with complex nutritional needs. NST improves the quality of treatment, clinical outcomes and reduces costs by avoiding unnecessary treatments and simplifying the treatments used. This study reviewed the outcomes achieved after the implementation of an NST on a group of patients who were given parenteral nutrition (PN) at a local acute hospital.

Methods A total of 81 patient records were retrieved for service evaluation analysis. Among the subjects, 44 of the patients on PN who were seen by the NST from Apr 2017 - Jun 2019 were compared to the historical control of 37 
patients on PN who have not received NST or dietitian intervention during the period from Mar 2016 - Mar 2017. Energy, protein, and micro-nutrients consumption adequacy were compared between the intervention and control groups. Patients without body weight and nutrition intake records were excluded. Independent t-test and chi-square test were used to compare the variables between both groups. A p-value of $<0.05$ was considered statistically significant.

Results The patients in the intervention group had a significantly higher energy and protein intake than that of the control group $(1279 \pm 353 \mathrm{kcal}$ versus $934 \pm 261 \mathrm{kcal} ; 58 \pm 16 \mathrm{~g}$ versus $43 \pm 12 \mathrm{~g}$ ). In terms of nutritional intake adequacy, the percentage of targeted energy and protein requirement achieved is significantly higher in the intervention group (81 $\pm 21 \%$ and $90 \pm 28 \%$ of their energy and protein requirements respectively), compared to $64 \pm 21 \%$ and $75 \pm 23 \%$ in the control group. Adequacy of micronutrient consumption is also significantly higher in the intervention group, $88.6 \%$ of patients in the intervention group achieved adequate intake of micronutrients from PN compare to $8.1 \%$ of patients in the control group.

Conclusions Nutrition care provided by the NST is promising to ensure the nutrition well-being of hospitalized malnourished patients. This review suggests that NST intervention can improve both macro- and micronutrient intake adequacies in patients on parenteral nutrition support. Further clinical outcomes studies on the benefits of NST are recommended.

\section{IDDF2021-ABS-0011 ENDOSCOPIC ULTRASONOGRAPHY IS USEFUL IN PREDICTING PERFORATION OF ENDOSCOPIC RESECTION FOR GASTRIC SUBMUCOSAL TUMORS ORIGINATING FROM THE MUSCULARIS PROPRIA}

Yi Lu*, Xianhua Zhuo, Jiachen Sun, Chujun Li. The Sixth Affiliated Hospital, Sun Yat-sen University, China

\subsection{6/gutjnl-2021-IDDF.105}

Background Predicting the risk of perforation during the endoscopic resection (ER) of gastric submucosal tumors (SMTs) originating from the muscularis propria (MP) could remind us to be more cautious and be prepared ahead of time. Moreover, we could select the appropriate patients for the inexperienced endoscopists. But for now, the predictive model was rare. Therefore, we conducted this study to figure out important parameters in the endoscopic ultrasonography (EUS) images to predict perforation, and try to build predictive models.

Methods From May 1st, 2013, to January 15th,2021, consecutive patients with gastric SMTs originating from MP and received ER in our hospital were retrospectively reviewed. The patients were classified into two groups based on whether they had perforation (the case group) or without (the control group). To evaluate the depth of the SMT locates using the quantification index, we came up with a parameter called the ratio of inner distance to outer distance (I/O ratio). Logistic multivariate analysis was used to identify potential variables and predictive models.

Results 199 EUS procedures (194 patients) were finally chosen for analysis. The median age of the patients was 53 years old, and $88(44.22 \%)$ were males. The en bloc resection rate had no difference in the two groups, while patients in the case group had longer procedure time, higher probability of infection, and longer postoperative hospital stay. Multivariate analysis showed that age, I/O ratio, and the pathology of the tumors were risk factors for perforation, and we further built two models to predict perforation, with an AUC of 0.836 (for Model 1) and 0.755 (for Model 2).

Conclusions EUS was useful in predicting perforation of ER for gastric SMTs originating from MP, and we have identified two models to predict perforation, which might serve as a useful tool in clinical practice.

\section{IDDF2021-ABS-0016 EFFICACY AND SAFETY OF PRE- ENDOSCOPIC TREATMENT FOR ASYMPTOMATIC OR INCIDENTAL STRICTURES IN CROHN'S DISEASE}

Junrong Chen*, Jiachen Sun, Yi Lu, Min Zhi. The Sixth Affiliated Hospital, Sun Yat-sen University, China

\subsection{6/gutjnl-2021-IDDF.106}

Background Current important treatment modality for stricture in Crohn's disease (CD) is endoscopic treatment. However, there is no unified treatment plan for asymptomatic or incidental strictures with CD patients (AIS).

Methods All eligible patients with stenosis of CD who were treated by endoscopic therapy from January 2013 to January 2021 were included (IDDF2021-ABS-0016 Figure 1. Endoscopic balloon dilation of small intestinal stricture with CD patients. A, Small intestinal stricture; B, Place $35 \mathrm{GW}$ through the stenosis segment; C, Dilation with $16 \mathrm{~mm}$ through-the-scope balloon; D, Post-dilation appearance). The primary outcomes were treatment success rate, post-procedural complications, endoscopic recurrence, additional surgical treatment rate, and stenosis-free survival with preendoscopic treatment for AIS or endoscopic treatment for symptomatic strictures.

Results A total of 87 patients of 125 procedures were studied, including pre-endoscopic treatment group $(\mathrm{N}=31)$ and endoscopic treatment group $(\mathrm{N}=56)$. The median follow-up time was 21 months (2-91 months). Cox multivariate regression analysis found that the type of treatment (HR 3.146, 95\%CI 1.081-9.156, $\mathrm{P}=0.036)$, balloon diameter (HR 3.877, 95\%CI 1.343-6.466, $\mathrm{P}=0.009$ ), total enteral nutrition (HR 2.946, 95\%CI 1.112-11.323, $\mathrm{P}=0.007$ ), and history of anal fistula surgery (HR 3.548, 95\%CI 1.412 10.648, $\mathrm{P}=0.032$ ) were risk factors for recurrent stenosis after endoscopic treatment. While duration (HR 5.860, 95\% CI 1.068-32.163, $\mathrm{P}=0.042$ ) and hypoproteinemia (HR 4.578 , 95\%CI $1.038-20.196, \mathrm{P}=0.045)$ were risk factors for additional surgery after endoscopic treatment. Compared with endoscopic treatment group, pre-endoscopic treatment group had the immediate technical success for therapy fairly (96.77\% vs. 91.07\%, P=0.573), lower endoscopic stenosis recurrence rate $(16.13 \%$ vs. $46.43 \%, \mathrm{P}=0.003)$, lower surgical treatment rate ( 0 vs. $16.07 \%, P=0.024)$, and higher 3 years stenosis-free survival $(85.9 \%$ vs. $50.4 \%)$. The postoperative complications in all 3 cases, bleeding in 1 case, 2 cases of perforation were in the endoscopic treatment group.

Conclusions CD patients with AIS were often inadvertently found during colonoscopy and might develop symptomatic 